\section{GENRE}

en séries
Genre en séries

Cinéma, télévision, médias

5 | 2017

Masculinités imag(in)les 2

\title{
Le trouble silencieux des hommes en série. La « masculinité en crise » dans les séries télévisées dramatiques nord-américaines centrées sur des personnages masculins
}

\section{Stéfany Boisvert}

\section{OpenEdition}

\section{Journals}

Édition électronique

URL : http://journals.openedition.org/ges/871

DOI : $10.4000 /$ ges.871

ISSN : 2431-6563

Éditeur

Presses universitaires de Bordeaux

Référence électronique

Stéfany Boisvert, «Le trouble silencieux des hommes en série. La « masculinité en crise » dans les séries télévisées dramatiques nord-américaines centrées sur des personnages masculins », Genre en séries [En ligne], 5 | 2017, mis en ligne le 01 juin 2017, consulté le 22 mars 2021. URL : http:// journals.openedition.org/ges/871 ; DOI : https://doi.org/10.4000/ges.871

Ce document a été généré automatiquement le 22 mars 2021.

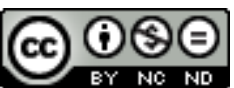

La revue Genre en séries est mise à disposition selon les termes de la Licence Creative Commons Attribution - Pas d'Utilisation Commerciale - Pas de Modification 4.0 International. 


\title{
Le trouble silencieux des hommes en série. La " masculinité en crise » dans les séries télévisées dramatiques nord-américaines centrées sur des personnages masculins
}

\author{
Stéfany Boisvert
}

1 Cet article propose une étude transnationale de la «masculinité en crise » en tant que norme représentationnelle dominante dans de nombreuses séries télévisées dramatiques contemporaines en Amérique du Nord. Bien qu'étant fort populaire aux États-Unis, particulièrement sur les chaînes câblées, cette thématique est loin de leur être exclusive et se retrouve également dans plusieurs séries canadiennes anglophones et québécoises francophones diffusées depuis $2005^{1}$. L'objectif de cet article est donc de parvenir à une connaissance élargie et approfondie d'une tendance narrative importante en Amérique du Nord, dans un contexte où la qualification des séries étatsuniennes en tant que productions "américaines" a souvent tendance à évacuer l'existence d'autres industries télévisuelles au sein de ce sous-continent.

2 Cet article s'appuie sur une analyse comparative préalablement réalisée concernant la représentation des hommes et la construction des discours sur la masculinité dans trente séries télévisées nord-américaines, principalement centrées sur des personnages masculins, et diffusées depuis 2005. Entre autres constats, cette recherche a ainsi révélé un attrait similaire pour la représentation de « masculinités en crise » au sein des trois aires culturelles étudiées (Québec, Canada, États-Unis). Comme il sera expliqué plus loin, la représentation de cette crise peut certes prendre diverses formes, mais elle renvoie généralement à une perception d'une déstabilisation des repères identitaires associés à la masculinité, ce qui aurait des conséquences négatives sur les parcours 
existentiels des hommes. À travers des références à quelques séries télévisées, cet article mettra ainsi en lumière les significations culturelles de cette tendance scénaristique et proposera trois grands modèles d'articulation de la «masculinité en crise " à la télévision: l'homme tourmenté, l'homme inadéquat et l'homme menacé. L'étude de ce phénomène culturel révèle que plusieurs significations se cachent derrière une telle tendance narrative, celle-ci pouvant même parfois servir, paradoxalement, de stratégie de légitimation des œuvres...et du genre.

\section{La thématique de la « masculinité en crise »}

De nombreux chercheur.e.s (Badinter, 1992 ; Clare, 2001 ; Faludi, 1999 ; Horrocks, 1994 ; Robinson, 2000; Rosin, 2010, 2012 ; Watson, 2009) témoignent d'une préoccupation centrale de nos sociétés contemporaines pour l'identité des hommes, perçue comme "en crise». De manière générale, cette théorie défend l'idée d'une "anxiété ontologique » (Shweder, 1994) de l'identité masculine depuis la fin du vingtième siècle. Les bouleversements socioéconomiques des dernières décennies, notamment l'impact des mouvements féministes, la "remise en question des rôles traditionnels" (Tremblay et L'Heureux, 2010: 99), l'entrée massive des femmes et de nouvelles communautés immigrantes sur le marché du travail et la contestation du patriarcat (Beynon, 2002: 159) ont remis en question, du moins partiellement, les conceptions traditionnelles de l'identité masculine et, dès lors, auraient provoqué une crise endémique du masculin. Cette vision est évidemment présentée de manière plus ou moins fataliste selon les chercheurs, certains y voyant tout simplement un état transitoire des identités de genre, d'autres une véritable tragédie ou une attaque en règle contre les hommes.

4 Certains spécialistes interprètent ainsi ce phénomène en postulant le caractère ontologiquement pathologique du genre masculin. Selon le psychothérapeute Roger Horrocks (1994), au sein de la civilisation occidentale, industrielle et capitaliste, la masculinité exigerait des hommes qu'ils portent un masque, un «faux soi », qu'ils souffrent silencieusement, qu'ils gardent le contrôle et qu'ils dissimulent leurs émotions. L'identité masculine entraînerait donc une forme d'autisme émotionnel (Horrocks, 1994) et de profonde détresse psychologique: "Leur état m'apparaît symptomatique d'un malaise de l'homme - ce que je nomme librement un autisme masculin - un sentiment d'être coupé de ses sentiments naturels, de son expressivité et des contacts avec les autres ${ }^{2} »$ (Horrocks, 1994 : 107). Plusieurs chercheurs font aussi allusion aux problèmes de violence et de criminalité, lesquels concernent davantage les hommes, en tant que preuves de cette dimension pathologique. Certains vont jusqu'à prophétiser la disparition imminente de la masculinité (MacInnes, 1998; Rosin, 2012) en tant qu'identité culturelle, qui serait devenue caduque ou fortement inadaptée et inadaptable aux sociétés contemporaines.

5 La plupart des tenants de cette thèse ont toutefois développé une théorie plus "élégiaque " (Heartfield, 2002): la masculinité y est alors perçue comme étant actuellement dans un état critique et ses défenseurs adoptent une position sympathisante à l'égard des hommes, considérés comme des «victimes de circonstances hors de leur contrôle $»^{3}$ (Heartfield, 2002: 2). Cette vision élégiaque renvoie habituellement à l'idée que la crise serait le signe d'une perte de repères identitaires, d'une anxiété nouvelle face à la signification de la masculinité, certains 
allant jusqu'à associer ce mal-être à une "féminisation » des hommes. Anthony Clare soutient ainsi que les hommes et leur identité seraient actuellement menacés, puisque les traits qui leur sont traditionnellement associés, en particulier la logique, la discipline, la maîtrise de soi, la rationalité et l'agressivité, seraient désormais critiqués et perçus comme des «stigmates de déviance » («stigmata of deviance») (Clare, 2001 : 68). La « crise de la masculinité » est aussi parfois spécifiquement interprétée comme une crise de la virilité (Courtine, 2011), alors que se répandraient chez les hommes une "obsession érectile»(10) et des angoisses multiples concernant leurs capacités sexuelles. Les mouvements masculinistes et mythopoétiques ${ }^{4}$, très en vogue depuis les années 1990, ont également contribué à véhiculer cette image d'hommes victimes, malheureux, dont l'identité est menacée, voire aurait été " perdue ».

6 Le fait de souligner l'omniprésence de cette vision de la masculinité ne revient toutefois pas à l'approuver, bien au contraire. Cette théorie prête en effet le flanc à de nombreuses critiques. Comme l'ont souligné Guionnet et Neveu, «le thème est gros d'un ensemble de simplifications, de questions mal posées » (Guionnet et Neveu, 2009: 338) et procède d'une généralisation, fortement réductrice et essentialiste (Guionnet et Neveu, 2009: 338; Whitehead, 2002: 55), qui gomme la diversité des identités masculines. En phase avec l'idéologie du genre enjoignant à toujours percevoir les hommes et les femmes selon un «dualisme oppositionnel» (Badinter, 1992), cette théorie suppose ainsi un renversement total des rapports de genre, qui nie la complexité des rapports sociaux ainsi que la persistance de pratiques discriminatoires envers les femmes, notamment l'inégalité dans l'accès à certaines positions socioprofessionnelles (Dupuis-Déri, 2012 : 91). Comme le résume Anthony Mac Mahon (2005), « la littérature sur la masculinité » tend à « s'approprie[r] de façon sélective les formes de féminisme dont les analyses négligent des aspects-clés des rapports de genre, en particulier lorsque celles-ci rendent invisibles les bénéfices matériels que les hommes retirent du patriarcat » (Mac Mahon, 2005 : 28-29).

7 Qui plus est, la masculinité a souvent été considérée « en crise » par le passé, et ce dans de nombreux pays. Aux États-Unis, les hommes ont été considérés «en crise » de manière récurrente depuis la fin du XVIII ${ }^{e}$ siècle (Kimmel, 2012; Lotz, 2014: 60). En France, les travaux historiques d'Annelise Maugue (1987) et d'André Rauch (2000) ont également documenté la thèse d'une "masculinité en crise ", attaquée et menacée par des forces "féminines ", dès la fin du XVIII ${ }^{e}$ siècle jusqu'en 1914. De telles recherches invalident la thèse d'une spécificité de l'époque actuelle, faisant naître le soupçon que la notion de crise serait inhérente au genre masculin lui-même, nécessaire à sa préservation. En effet, cette théorie est souvent réactivée lors de périodes de transformations sociales, qui donnent lieu chez certains groupes à des tentatives de revalorisation d'identités traditionnelles et conservatrices (Kimmel, 1987: 123). Constatant cette récurrence du thème, Judith Allen (2002) se demande si les hommes ne seraient pas « interminablement » en crise. Bien évidemment, le fait de critiquer cette théorie ne revient pas à contester l'existence de certaines problématiques touchant majoritairement les hommes, mais à promouvoir une vision plus complexe et déconstructiviste des catégories genrées. L'idéologie du genre est construite sur une vision binaire et oppositionnelle.

8 La thématique de la "masculinité en crise» est récurrente dans les fictions contemporaines, ce qui rend nécessaire son analyse, non seulement pour la déconstruire, mais aussi pour comprendre quelles fonctions narratives elle revêt au 
sein des séries. La «masculinité en crise » doit en ce sens être considérée comme un acte performatif (Robinson, 2000; Walsh, 2010), une tendance interprétative et représentationnelle centrale au sein des récits télévisuels contemporains, qui véhicule une vision dominante des rapports de genre au XXI ${ }^{\mathrm{e}}$ siècle.

\title{
La « crise masculine » dans les séries télévisées états- uniennes
}

\author{
« Je crois que je ne l'avais jamais réalisé avant, \\ mais ça doit aussi être difficile d'être un \\ homme. $»^{5}$ \\ (Rachel, Mad Men, S01xE01)
}

9 Aux États-Unis, le tournant du siècle a été caractérisé par l'arrivée en force à la télévision de personnages masculins «en crise ». Une telle vision du genre se traduit généralement par une investigation poussée du mal-être de certains personnages masculins, de leur sentiment de culpabilité endémique ou d'inadéquation, de leurs angoisses existentielles ou de leur détresse psychologique. La chaîne de basic cable ${ }^{6}$ AMC doit notamment sa notoriété à cette mise en scène empathique d'hommes en crise. Mad Men (AMC, 2007-2015) se déroule dans une compagnie de consultants en publicité des années 1960 à New York, et explore la mélancolie et les bouleversements identitaires de Don Draper (Jon Hamm), le personnage principal, un homme hanté par une enfance difficile et par le lourd secret de son identité d'emprunt: vivant une existence insatisfaisante, Don a décidé, pendant son engagement militaire en Corée, de changer d'identité avec un soldat décédé, pour être finalement confronté à la réalité douceamère d'une existence où ses tourments personnels ne le quitteront jamais. Quelques flashbacks viennent souligner les moments douloureux ou les pensées mélancoliques de Don, mettant ainsi en lumière cette vision d'une masculinité tourmentée que le masque du prestige social ne réussit à camoufler que temporairement. Malgré de grandes différences scénaristiques entre ces deux séries, Breaking Bad (AMC, 2008-2013) peut également être perçue comme une fiction dont l'efficacité dramatique repose sur l'exploitation du modèle initial d'une "masculinité en crise", d'un homme qui deviendra « méchant » (Jost, 2015), notamment par désir d'être enfin reconnu dans son identité de genre. Breaking Bad est l'histoire de Walter White (Bryan Cranston), un père quinquagénaire professeur de chimie dans un lycée d'Albuquerque. Après avoir appris qu'il était atteint d'un cancer du poumon inopérable, il décide de fabriquer de la méthamphétamine (crystal meth) afin de subvenir aux besoins financiers de sa famille après sa mort. Durant l'épisode pilote, Walt est représenté comme un homme non reconnu en tant qu' "homme » et manquant d'assurance. Il est ridiculisé à plusieurs reprises et accusé de ne pas agir « comme un homme ». En ce sens, Breaking Bad exploite cette vision actuelle d'une masculinité en péril et malheureuse. Et de même que Walter White se lancera entre autres dans la production de méthamphétamines pour se sentir puissant et «vivant» [S05XE16] et enfin un «homme», le personnage principal de Fargo (FX, 2014-), Lester, tuera sa femme après que celle-ci l'ait provoqué en lui disant... qu'il n'était pas un homme!

Oscillant entre le registre dramatique et la comédie noire, la première saison de la série Fargo débute au moment de l'arrivée, dans une petite ville du Minnesota, de Lorne Malvo (Billy Bob Thornton), un criminel qui exercera une influence néfaste sur Lester 
Nygaard (Martin Freeman), un vendeur d'assurances. La série se concentre sur les mésaventures de ces deux protagonistes, ainsi que sur les deux policiers qui enquêtent sur une série de meurtres. Tout comme dans Breaking Bad, dès les premières minutes de l'épisode pilote, Lester est présenté comme un homme timide, maladroit, méprisé par son épouse ainsi que par certains habitants de la ville. L'importance de la dimension genrée dans la crise que vit Lester est soulignée quand il tente, en vain, de réparer sa machine à laver. Devant la réaction méprisante de son épouse Pearl, Lester tente de se défendre en affirmant avoir voulu agir comme un homme, ce que celle-ci conteste aussitôt: "Mais tu n'es pas un homme, Lester. Tu n'es même pas la moitié d'un homme. $»^{7}$ [S01XE01] Lester la tue alors d'un coup de marteau sur le crâne. Au moment de commettre ce meurtre, la musique dramatique qui contraste avec le ton plus comique des scènes précédentes, suggère la gravité de l'acte commis mais aussi, d'une certaine façon, celle de la blessure symbolique et narcissique ressentie par Lester face à son identité niée et méprisée.

11 Les personnages masculins de ces deux séries sont ainsi représentés comme étant « en crise ", c'est-à-dire victimes de souffrances émotionnelles, d'ostracisme et d'un sentiment persistant d'inadéquation, non pas lorsqu'ils commettent des crimes atroces, mais bien avant de les commettre, autrement dit lorsqu'ils sont punis pour leur nonconformité aux normes de genre.

12 Autre poids lourd de l'industrie télévisuelle câblée aux États-Unis, la chaîne FX a elle aussi construit sa réputation en proposant des contenus plus violents et plus complexes (Curtin et Shattuc, 2009: 133-134), et des récits souvent peuplés de protagonistes masculins complexes, moralement ambigus et tourmentés (The Shield (FX, 2002-2008), Nip/Tuck (FX, 2003-2010), Rescue Me (FX, 2004-2011), etc.). Par exemple, la série Rescue $\mathrm{Me}$, produite quelques années seulement après le 11 septembre 2001, aborde le quotidien de pompiers de New York qui ont été appelés à intervenir durant la plus grande tragédie de l'histoire récente des États-Unis et ont été confrontés à la mort de plusieurs collègues. Tommy Gavin (Denis Leary), le personnage principal, est habité par des images obsédantes et traumatiques, voyant autour de lui les personnes qu'il n'a pu sauver lors de différents incendies, ainsi que son cousin mort durant les attentats. La série illustre ainsi la crise du personnage, mais aussi le lourd sacrifice de ces hommes qui acceptent d'affronter quotidiennement la mort et de multiples dangers afin de protéger la collectivité. Maugréant parfois contre le manque de reconnaissance de la société à leur endroit, rappelant la dévalorisation des hommes dans un contexte de revendications féministes constantes, les protagonistes de Rescue Me sont représentés comme étant coincés entre une conception traditionnelle de la masculinité, qui les enjoint au stoïcisme, et un besoin d'exprimer leurs émotions et leurs souffrances. Et lorsqu'ils essaient d'exprimer ces émotions négatives, ils sont souvent rabroués par des femmes qui affichent un désintérêt complet à leur égard. Lorsque Tommy se confie à Sheila, l'épouse de son cousin décédé, et lui parle de ses problèmes familiaux, elle le traite de "pussy", l'enjoignant à se battre plutôt qu'à se plaindre : "Montre que tu as des couilles! $»^{8}$ [S01XE03]. Les protagonistes de cette série apparaissent ainsi doublement en crise: confrontés à des conflits identitaires et des expériences traumatiques, ils sont abandonnés par une société qui refuse de les entendre.

Des œuvres plus récentes des chaînes câblées premium, comme la série d'enquêtes True Detective (HBO, 2014-), peuplée d'hommes taciturnes en quête de rédemption, ou encore Ray Donovan (Showtime, 2013-), misent aussi sur la représentation de masculinités 
tourmentées, en proie à de profondes crises existentielles. Ray Donovan est, ironiquement, un gestionnaire de crises pour nantis ; avec ingéniosité, violence et peu de mots, il travaille à sauver les autres, y compris les membres de sa propre famille, tout en tentant de fuir un passé traumatique à travers une consommation parfois excessive d'alcool. Derrière une posture stoïque et taciturne, Ray cache donc lui aussi une grande vulnérabilité et une douleur existentielle: abusé sexuellement par un prêtre durant son enfance, sans parents pour prendre soin de lui, cet homme porte aussi le fardeau de la culpabilité de la mort de sa sœur qui s'est jetée du haut d'un immeuble. Dans de nombreuses séries telles que Ray Donovan, l'utilisation de séquences imaginées/oniriques ou de flashbacks permet ainsi la mise en images métaphorique ou silencieuse du vécu des hommes, de leurs traumas, de leurs pensées mélancoliques et négatives. Lors d'une séquence onirique de l'épisode pilote de Ray Donovan [S01XE01], Ray s'endort sous l'effet de l'alcool. Son rêve est alors mis en images à travers une séquence résumant les divers événements négatifs de sa vie : le décès de sa mère, l'abus sexuel dont il a été victime et le suicide de sa sœur.

Depuis quelques années, certains chercheurs ont commencé à documenter cette centralité de la thématique de l'homme "en crise» ou dépressif dans les séries télévisées états-uniennes (Baker, 2015 ; Lotz, 2014 ; Martin, 2013 ; Mittell, 2015 ; Rehm, 2014 ; Sérisier, Boutet et Bassaget, 2011 ; Shimpach, 2010). Toutefois, il est rarement souligné que plusieurs séries canadiennes et québécoises ont elles aussi fait preuve d'un intérêt similaire pour cette tendance représentationnelle, ce qui en fait une récurrence narrative beaucoup plus transnationale.

\section{La « crise masculine » dans les séries télévisées canadiennes}

Bien qu'occupant une place moins centrale que dans la programmation états-unienne, l'exploration des «masculinités en crise " prend plus d'importance depuis la dernière décennie à la télévision canadienne-anglaise. À titre d'exemple, le récit de la populaire série policière Flashpoint (CTV, 2008-2012) qui raconte le quotidien de l'unité stratégique d'intervention ${ }^{9}$ de la ville de Toronto, débute au moment où Ed (Hugh Dillon), le personnage principal, est obligé d'abattre un homme armé qui a pris une femme en otage. En fier représentant de la gent masculine, Ed tente évidemment de cacher son désarroi à la suite de cet acte, mais des effets visuels et sonores permettent aux téléspectateurs de deviner le trouble silencieux qui le hante. Ainsi, à la fin de la journée, tous les policiers de l'unité d'intervention se retrouvent dans un bar. Les discussions entre collègues y sont animées. La caméra effectue une légère rotation autour des personnages, suggérant la dynamique enjouée du groupe et leur solidarité. Mais au moment où la caméra cadre Ed, le son intra-diégétique est soudain étouffé, suggérant le trouble intérieur du personnage et son incapacité à interagir. Ed regarde alors en direction du téléviseur qui diffuse un reportage sur l'homme qu'il vient d'abattre. Le son intra-diégétique n'est rétabli qu'au moment où Ed quitte la pièce, comme un signe que le trauma du personnage principal le coupe du monde. Une telle prémisse est représentative de ce qui est de plus en plus perçu aujourd'hui comme une amorce narrative efficace, à savoir la révélation de la complexité émotionnelle et mélancolique des hommes " héros du quotidien ». Dans un épisode marquant de cette même série, Ed sera appelé à intervenir auprès d'un ancien collègue en proie à une 
grave dépression, qui menace de se suicider [S01XE12]. Le monologue d'Ed, alors qu'il tente de le consoler, résume cette vision d'une masculinité sacrifiée au bien collectif: «Tu sais ce que je vois d'autre? Tous ces appels auxquels nous n'avons pas répondu à temps. [...] Tu n'es pas le seul à voir des fantômes, Danny. $»^{10}$ [S01XE12] Lorsque Ed réussit enfin à convaincre son collègue de poser son arme, les deux hommes s'étreignent et pleurent à chaudes larmes, révélant ainsi publiquement le poids insoupçonné de l'identité masculine. Dans un registre similaire, la série policière Cracked (CBC, 2013) est centrée sur Aidan (David Sutcliffe), un enquêteur au sein d'une unité psycho-criminelle, anciennement spécialiste en intervention d'urgence, qui est atteint de stress post-traumatique après avoir accidentellement tué un garçon lors d'une intervention. Au-delà de l'enquête différente proposée à chaque épisode de cette série, il est question, en filigrane, du cheminement tortueux du protagoniste jusqu'à sa décision de demander une aide psychologique. «Il n'est pas détruit, mais simplement abîmé ${ }^{11}$ » affirmera Danielle, sa collègue psychiatre, interprétant les comportements erratiques de son homologue masculin comme un véritable appel à l'aide.

Le succès critique Durham County (The Movie Network, 2007-2010) met également en opposition, dans sa première saison, deux hommes confrontés à des crises différentes. Cette série dramatique est centrée principalement sur le personnage de Mike Sweeney (Hugh Dillon), un policier taciturne, assailli par le malheur; affecté aux enquêtes criminelles, il doit élucider le meurtre de plusieurs jeunes femmes, ce qui l'amène à enquêter sur le meurtre de sa maîtresse et le confronte à un deuil inavouable, ainsi qu'à des sentiments conflictuels vis à vis de sa femme qu'il a trompée alors qu'elle combattait un cancer. De son côté le tueur en série, Ray Prager (Justin Louis), un ancien ami de Mike, était promis à un brillant avenir en tant que joueur de hockey professionnel, mais a dû faire le deuil de cette carrière à la suite d'un accident de voiture. Lorsque Ray s'en prend à des femmes, il est représenté comme un homme cherchant à prouver son hégémonie ${ }^{12}$, à travers son ascendant et son contrôle absolu sur la gent féminine qui ne lui accorde pas l'intérêt souhaité. Son comportement s'apparente à celui qu'analyse Susan Faludi (1999) à propos des hommes qui se sentent trahis par la société contemporaine et n'acceptent pas la perte du statut enviable qui leur avait été promis. La série propose donc directement, à travers non seulement le personnage de Ray mais également celui de Mike, qui a lui aussi parfois des comportements violents et misogynes, une représentation tragique de la masculinité et des conséquences néfastes de la socialisation au genre.

\section{Les séries québécoises et la « condition masculine »}

17 Encore plus présente que dans la production canadienne-anglaise, la thématique de l'homme « en crise » est devenue une tendance centrale de la télévision québécoise, et surtout de son réseau public Ici Radio-Canada Télé. Centrée sur le quotidien de trois portiers d'un bar de Montréal, Minuit, le soir (Radio-Canada, 2005-2007) explorait sans pudeur, mais avec beaucoup de compassion, la vie intime de ses trois protagonistes: Gaétan (Julien Poulain), un quinquagénaire confronté à la solitude et qui cache aux autres sa honte d'être analphabète; Louis (Louis Champagne), un homme sensible souffrant de problèmes érectiles; Marc (Claude Legault), enfin, un ancien soldat de la marine militaire aux tendances dépressives qui cherche à se départir de ses comportements violents. Malgré la grande complicité entre les trois hommes, la mise 
en scène du réalisateur Podz (Daniel Grou) octroie une large place aux flashbacks et autres stratégies narratives qui permettent d'exposer les non-dits, les émotions, les tourments qui contrastent souvent avec la performance publique de la masculinité. La plus récente série policière chapeautée par le même réalisateur, 19-2 (Ici Radio-Canada Télé, 2011-2015), poursuit une exploration similaire de héros du quotidien dont la vie intime est marquée par de multiples crises. L'un des protagonistes, Nick Berrof (Réal Bossé), est un homme animé d'un grand sens de la justice, mais qui porte constamment le poids d'une existence en crise. En guerre contre lui-même, habité de tendances violentes et de nombreux "démons", Berrof se transformera progressivement en justicier sanguinaire; cherchant à venger un ami et à mettre fin aux activités d'un réseau pédophile, il provoquera le malheur et la mort autour de lui avant d'être finalement arrêté par la police ${ }^{13}$. La masculinité semble ainsi constamment mener à la crise ; si d'aventure le bonheur ose s'installer, il est rapidement ravi à l'homme dans un moment de revirement dramatique, tel Marc Forest dans Minuit, le soir, dont la promesse d'union avec la femme qu'il aime n'aura été qu'un mirage, puisqu'il sera abattu au moment de la rejoindre. Dans un registre plus comique, la série Les Invincibles (Radio-Canada, 2005-2009) s'attarde sur le vécu tortueux de quatre hommes ayant fait le pacte de rompre avec leur conjointe respective afin d'accéder à une vie plus heureuse, selon eux, et plus conforme à la culture masculine. La série propose ainsi indirectement une vision de la "masculinité en crise", souvent perçue comme le résultat d'une société post-féministe où l'égalité hommes-femmes serait atteinte, ce qui rendrait caduc le mouvement féministe (Dupuis-Déri, 2015 ; Modleski, 1991 ; Tasker et Negra, 2007: 1). Dans ce contexte, les hommes sont souvent confrontés à des sentiments persistants d'inadéquation, en mal de modèles positifs et soumis à des femmes accomplies qui ne semblent pas vivre de tels paradoxes identitaires ${ }^{14}$.

\section{Une typologie des hommes en crise}

18 Une approche herméneutique, centrée sur le récit global, long et sériel, des personnages masculins des séries mentionnées révèle ainsi souvent des parcours identitaires négatifs où prédominent les dépressions, tourments, désertions, fautes, trahisons, humiliations ou sentiments d'inadéquation. Plus spécifiquement, en tant que pulsion narrative, la crise peut donner lieu à trois grands modèles de masculinité : l'homme tourmenté, l'homme inadéquat et l'homme menacé. La première catégorie concerne tous ces protagonistes dont l'identité et le parcours existentiel sont marqués par une dominante émotionnelle négative, une "crise intérieure » $(E d w a r d s, 2006)^{15}$ : c'est l'homme en proie au mal de vivre, parfois traumatisé par un lourd passé. La popularité des récits télévisuels où l'on sonde l'âme de héros masculins tourmentés ou profondément mélancoliques renvoie à cette catégorie, de même que les protagonistes de séries policières ou médicales qui, portant le fardeau de trop nombreuses tragédies humaines, sont en proie à une profonde souffrance empathique. La crise peut évidemment varier en intensité, passant du simple marasme émotionnel à la récurrence ponctuelle d'un traumatisme jusqu'à la plus grave des dépressions.

De son côté, l'homme inadéquat est présent dans toutes ces séries où le protagoniste se considère lui-même ou est perçu par les autres comme possédant une identité de genre problématique, ridicule ou inadaptée à son environnement: il s'agit de l'homme immature, l'inadapté social ou perçu comme étant «trop féminin». La crise peut 
également être abordée dans ses dimensions extérieures à travers tous ces récits où l'homme est représenté comme étant menacé, attaqué, ouvertement dévalorisé, et dès lors comme étant la principale victime des récentes transformations socioéconomiques. Dans ce dernier type de récit, l'homme menacé, en tant que victime, peut parfois se transformer en véritable " angry white man » (Kimmel, 2013) - songeons à Breaking Bad se révoltant contre un état de crise ou la perte d'un statut privilégié. Ces modèles de masculinité peuvent également se combiner au sein d'une même fiction. En tant que ressort dramatique, la crise peut aussi être utilisée à divers stades du récit : en tant que prémisse, en tant qu'élément plus ou moins constant de l'identité d'un personnage, ou encore comme achèvement malheureux de son parcours. Il s'agit ainsi d'un ressort narratif polyvalent, mais qui témoigne dans tous les cas d'une fascination pour une identité masculine profondément déstabilisée.

\section{Les séries télévisées contemporaines face au discours de la « masculinité en crise »}

20 Le caractère fictionnel de ces séries télévisées ne doit pas empêcher de reconnaître la dimension interprétative de ces «mondes possibles » (Doležel, 1998) en regard de notre réalité sociale. Comme l'a souligné Edgar Morin, la dominante narrative des récits de la culture de masse peut être révélatrice d'une certaine " conception articulée de la vie " (2008: 104). Dans cette perspective, la dominante négative et le rejet quasi systématique du happy end dans les récits centrés sur des hommes peuvent être considérés comme des dimensions centrales du mythe de la masculinité à la télévision contemporaine, et non comme de simples « coïncidences » narratives. Selon Éric Macé (2006), la télévision peut en effet être conçue comme un «double du monde social» révélateur des visions du monde et rapports sociaux. Cette situation permet d'expliquer la présence récurrente de l'homme en crise en tant que tendance télévisuelle transnationale.

21 Certes, la thématique de la "crise ", si présente dans les séries, relève aussi d'une nécessité narrative. Comme les recherches de Sabine Chalvon-Demersay (1994) l'ont mis en lumière, une fiction doit se construire à travers la dramatisation de conventions, et dès lors s'élaborer autour d'une crise. Plus encore, le contexte socioculturel est fréquemment interprété comme le théâtre d'une crise polymorphe ou d'un passage d'une "mythologie du bonheur " à une "problématique du bonheur» (Morin, 1975 : 143): on parle ainsi d'une crise des identités, du lien social, du lien familial, des institutions...

Certes, la complexité et l'indétermination grandissantes des sociétés contemporaines ont favorisé l'avènement de "néo-séries " (Perreur, 2007, 2011) caractérisées par un "réalisme pessimiste» (Perreur, 2011: 103) et des personnages confrontés à de multiples crises. En ce sens, sans nier les différences culturelles, l'imaginaire télévisuel français contemporain analysé par Chalvon-Demersay, caractérisé par la «thématique souterraine de la crise " et la "coloration foncièrement pessimiste" (ChalvonDemersay ${ }^{16}$, 1994: 42) des récits, possède des similitudes avec d'autres corpus nationaux. Et pourtant, loin d'être la simple illustration de destins individuels asexués, cette conception d'une crise sociale est associée de manière préférentielle, à la télévision, aux hommes blancs hétérosexuels, particulièrement ceux de la génération des 30-50 ans, en tant que catégorie ébranlée plus que d'autres par les récentes 
mutations sociales, culturelles et économiques. Les protagonistes féminines peuvent certes être aux prises avec des problématiques existentielles; toutefois, de manière générale, les hommes y sont beaucoup plus souvent représentés comme malheureux, ayant une existence jalonnée de tourments et de problèmes existentiels souvent insolubles. Compte tenu de cette polarisation des cheminements masculins et féminins, ce que les séries télévisées mettent en récit est donc prioritairement une «crise de la masculinité", non pas une crise du genre ou une simple représentation des "problèmes" ponctuels des hommes. Cette thématique, en tant que représentation hégémonique des hommes blancs hétérosexuels dans le contexte économique néolibéral (Baker, $2015:$ 1), peut donc être interprétée comme une forme exemplaire de « conformisme provisoire de la programmation » (Macé, 2003, 2006). Certains créateurs revendiquent d'ailleurs ouvertement cette thématique.

À titre d'exemple, en 2010, après la publication de l'article «The End of Men » signé par la journaliste Hanna Rosin pour The Atlantic, plusieurs professionnels de l'industrie télévisuelle ont fait référence à ce texte en tant qu'inspiration. Matière première d'un livre publié deux ans plus tard (Rosin, 2012), cet article défend l'idée que la société contemporaine marquerait la fin du règne hégémonique des hommes et la montée en puissance des femmes, dont les traits de personnalité (l'écoute attentive, l'ouverture à l'autre, la communication, etc.) les rendraient beaucoup plus adaptées à notre société postindustrielle. Selon un directeur de programmation de CBS, au moment de produire la saison télévisuelle suivant la parution de cet article, vingt producteurs de sitcoms y auraient fait directement référence, affirmant que celui-ci faisait état d'une nouvelle réalité sociale qu'il fallait absolument représenter sur le petit écran (Chozick, 2011 ; Rosin, 2011). De même, au Québec, les créateurs de la série 19-2 ont exprimé leur intérêt pour cette vision particulière du masculin. Comme l'a mentionné une de ses auteurs, Joanne Arseneau : «En fait, ce qui m'intéressait le plus, c'était de rendre compte d'une masculinité en péril à travers ces deux hommes blessés " (Petrowski, 2011). 19-2 a d'ailleurs récemment fait l'objet d'une adaptation au Canada anglais pour la chaîne Bravo (2014-) ; bien que les deux versions proposent des interprétations distinctes des masculinités locales (Boisvert, 2016), cette thématique de la crise des hommes est incontestablement une des facettes de l'œuvre qui a attiré les diffuseurs.

\section{Interpréter la masculinité en crise}

Selon Sally Robinson, «la rhétorique de la crise est suffisamment flexible pour accommoder une diversité de récits ${ }^{17}$ qui ne relèvent pas tous d'intentions similaires (2000: 11). Non seulement la crise peut donner lieu à différents modèles de personnages, comme nous l'avons montré, mais mettre en scène des hommes en proie à des difficultés existentielles peut également servir de multiples fins et être interprété de différentes façons ${ }^{18}$ : cette "crise» peut servir à révéler les dangers de certains aspects de la socialisation au genre - ainsi en est-il des hommes de Durham County, socialisés à la violence et à la domination, ou encore des critiques adressées par la fille de Mike, Sadie, concernant la culture du viol encouragée par la société contemporaine -, ou encore les souffrances des hommes enfermés dans le carcan d'une identité restrictive ${ }^{19}$; encourager une transformation des identités masculines ou, au contraire, se focaliser sur la crise existentielle, perçue comme endémique à la masculinité ; ou encore, revendiquer le statut de victime pour les hommes à la suite 
d'une transformation des genres qui serait "allée trop loin » (Dupuis-Déri, 2015), provoquant souffrance et dissolution des identités et des couples hétérosexuels. Comme le rappellent Proulx et Bélanger, le concept de crise est fortement polysémique : «L'écriture chinoise traduit le concept de crise par deux pictogrammes signifiant simultanément 'danger' et 'occasion à saisir'. » (2001:29) Ce terme apparaît donc particulièrement adapté pour une mise en scène de visions contemporaines du genre, travaillées par une conflictualité de plus en plus grande de discours hégémoniques et contre-hégémoniques (Maigret et Macé, 2005).

Certains récits de la crise masculine peuvent être interprétés comme une réactualisation d'un discours masculiniste et antiféministe, à travers une vision victimisante des hommes. Nous définissons le masculinisme comme « un mouvement social conservateur ou réactionnaire qui prétend que les hommes souffrent d'une crise identitaire parce que les femmes en général, et les féministes en particulier, dominent la société et ses institutions" (Dupuis-Déri, 2015: 43). Plus fondamentalement, le mouvement masculiniste véhicule souvent l'idée que "le-féminisme-est-allé-troploin » (Dupuis-Déri, 2012 : 97 ; Blais et Dupuis-Déri, 2015) et « s'oppos[e] à l'égalité de genre » (Welzer-Lang et Zaouche Gaudron, $2011: 8$ ), prônant une égalité restrictive qui doit préserver la différence à tout prix. Bien que pouvant être traversées d'idéologies contradictoires, certaines séries (Rescue Me, Les Invincibles, etc.) semblent en effet véhiculer parfois cette image d'un monde où les hommes sont les grands perdants face à des femmes, sinon plus heureuses, du moins plus indépendantes, voire manipulatrices ou insensibles à leurs problèmes. Le cas du personnage de Lyne (surnommée Lyne-lapas-fine) dans Les Invincibles, femme autoritaire et castratrice face à son conjoint Carlos, pourrait ainsi être considérée comme un parangon de cette vision d'une société valorisant systématiquement les intérêts dits "féminins", où les femmes sont professionnellement accomplies, savent ce qu'elles veulent et sont en contrôle. Le fait que les quatre protagonistes masculins de la série créent initialement le pacte de rompre avec leur conjointe respective en scandant «Couilles et solidarité! » témoigne de cette vision d'une société où la norme sociale de la monogamie et de l'engagement conjugal est prioritairement associée aux femmes. Les hommes peineraient ainsi à trouver leur place. De même, dans Rescue Me, l'indifférence de plusieurs femmes face aux problèmes psychologiques des hommes tend à véhiculer cette image d'un monde insensible à la condition masculine et dévalorisant pour les hommes. Dans la première saison, Kenneth, un membre de la caserne, demandera à sa conjointe Phyllis de lire les poèmes qu'il a écrits afin de surmonter son choc post-traumatique. Consterné, il découvrira que celle-ci a honte de lui et refuse d'entendre sa souffrance [S01XE03]. Plus généralement, il est manifeste que la popularité transnationale de cette thématique tend à véhiculer la vision d'un monde post-féministe, au sein duquel les objectifs du féminisme ayant désormais été atteints, la nouvelle liberté et l'égalité des femmes se seraient retournées contre la gent masculine et auraient fait des hommes les grandes victimes de la société d'aujourd'hui. Cette vision laisse notamment sous-entendre que l'identité des femmes relèverait quant à elle de l'évidence: si les hommes ne savent plus ce qu'ils sont ou doivent être, c'est que les femmes, elles, ne le sauraient que trop bien!

Toutefois, dans la plupart des séries, la crise des personnages masculins nous semble plutôt servir à complexifier leur identité narrative, et dès lors à représenter d'une manière idéologiquement ambivalente les conséquences d'une "rupture d'équilibre " 
des modèles traditionnels de genre (Dubar, 2010). Le fait que les hommes soient fréquemment malheureux ne doit donc pas nécessairement être interprété de manière négative, puisqu'il témoigne aussi d'une transformation des scripts identitaires de genre et d'une valorisation plus importante de la sensibilité masculine. Selon Jason Mittell, la représentation récurrente de «masculinités blessées » (damaged masculinity) peut être interprétée comme une tendance progressiste qui brouille les distinctions binaires du genre en associant les hommes à des tendances narratives et à des thématiques à la fois "féminines» et "masculines" (2015: 252). La crise des personnages masculins est en effet rendue possible par une hybridation de genres narratifs distincts, notamment une utilisation beaucoup plus répandue des codes du mélodrame (représentation accrue de l'intimité, de la sphère privée, de l'émotivité) dans des genres télévisuels traditionnellement qualifiés de "masculins», tels que les séries policières, criminelles ou médicales. Dans une étude sur la genrification ${ }^{20}$ des modes narratifs traditionnels de la télévision, John Fiske affirmait d'ailleurs que les formes "féminines", telles que les soap operas, sont caractérisées par une ouverture narrative, autrement dit une feuilletonisation pouvant ne jamais connaitre de conclusion, ainsi que par le dévoilement de l'intimité et des sentiments (2011 [1987]: 217). En ce sens, la feuilletonisation de la quasi-totalité des séries abordées ici ainsi que l'importance dévolue à la sensibilité des hommes permettent de valoriser leur identité émotionnelle, plutôt que simplement professionnelle, d'explorer longuement leurs conflits existentiels, s'opposant ainsi aux codes traditionnels masculins de la résolution rapide des conflits (Fiske, 2011) et d'une représentation de personnages rationnels et stoïques. Par exemple, Ray Donovan met certes en scène un homme fort, violent, adultère, musclé et impétueux, parangon d'une norme masculine, mais le récit est complété par une exploration empathique des émotions et de la sensibilité de cet homme dans la sphère privée, surtout lorsqu'il se retrouve seul dans son appartement en ville. Durham County récupère de son côté le genre policier, traditionnellement qualifié de masculin (Feasey, 2008) et met en scène une violence inouïe; toutefois, la narration s'attarde davantage sur les émotions et le vécu personnels des protagonistes plutôt que sur l'action, venant encore ici complexifier des modèles de masculinité hégémonique. De même, au Québec, le feuilleton sérialisant (Benassi, 2004) 19-2 amalgame le genre narratif de la série policière avec ceux du mélodrame et du téléroman (une forme fictionnelle propre au Québec), traditionnellement qualifiés de "féminins ». Les hommes y apparaissent donc très sensibles, vulnérables, voire souvent davantage que leurs collègues féminines.

Selon Amanda D. Lotz (2014: 88), dans les nouveaux récits de "crise masculine » aux États-Unis, les femmes ne sont habituellement pas représentées comme les grandes responsables; il faut donc éviter d'interpréter ces récits négatifs comme le signe systématique d'une réaction violente et accusatrice (backlash) contre les femmes ou le féminisme. Hormis quelques exceptions que nous avons présentées, cette conclusion s'applique en effet aux séries que nous avons étudiées. Le rappel souvent explicite de normes du genre masculin - les hommes sont fréquemment rappelés à leurs responsabilités en tant qu'hommes, questionnés dans leur masculinité, enjoints à « être des vrais hommes" - permet de corroborer cette vision populaire de la condition masculine, tout en évitant de tenir des propos ouvertement antiféministes ${ }^{21}$. En déconstruisant le stéréotype de l'homme fier et fort, toujours égal à lui-même, les fictions télévisées cherchent à complexifier les normes de genre; par là-même, elles 
sont des tentatives de construction de nouveaux modèles de masculinité (Feasey, 2008 ; Lotz, 2014).

Et pourtant, tout en reconnaissant qu'il s'agit là de stratégies de négociation de genre, il nous semble devoir aller plus loin. Central en cultural studies, le terme de « négociation » est certes adéquat pour définir ces nouvelles représentations, mais il ne met pas en lumière les forces contradictoires à l'œuvre au sein d'une même performance de genre, ou des visions conservatrices moins visibles. Selon Mélissa Blais, la culture populaire tente en effet fréquemment de construire des représentations qui relèvent d'une forme de négociation "entre le féminisme et l'antiféminisme sur fond d'idéologie patriarcale $»^{22}$ (2015: 104). Si nous reprenons cette logique, dans les séries analysées, la majorité des récits peuvent s'interpréter comme des stratégies obliques de valorisation du genre et de la masculinité elle-même. Loin du discours unilatéralement négatif, la popularité de mâles torturés et affligés peut en effet être une stratégie narrative de valorisation de ces masculinités imaginées, lesquelles apparaissent complexes, intéressantes et tellement "masculines " précisément parce qu'elles ne sont pas heureuses. De tels récits peuvent notamment être interprétés comme une réactualisation de l'archétype du héros romantique associé à la masculinité. Dans plusieurs cas, le malheur des personnages est d'autant plus valorisant qu'il met en lumière la dimension sacrificielle ou idéaliste des hommes acceptant souvent d'endurer une existence malheureuse pour le bonheur des femmes, des enfants, de la collectivité, voire de l'humanité. En d'autres termes, la représentation récurrente des chocs posttraumatiques des hommes dans les séries policières permet certes une complexification de leur identité, mais elle permet également de représenter, de manière positive, leur dévouement et leur héroïsme. La série policière SouthLAnd (NBC, TNT 2009-2013) se conclut ainsi tragiquement par une illustration du sacrifice et de l'existence malheureuse de John (Michael Cudlitz), l'un des personnages principaux : à la suite d'un choc post-traumatique (il a été pris en otage par deux toxicomanes alors qu'il était en service et a été témoin du meurtre de son collègue) et de déboires personnels, John se trouve en proie à une grande détresse psychologique ; un soir, il attaque physiquement un voisin antipathique, ne semblant pas trouver d'autre issue à son mal-être. Il est alors abattu par la police, la série se clôturant sur cette image d'un homme "attaqué » (sans doute tué) par ses propres collègues [S05XE10] ${ }^{23}$. Dans un autre ordre d'idées, la psychologisation de la "crise masculine " peut aussi parfois servir à nier ou, du moins, à relativiser l'hégémonie de certains personnages par un rappel de leurs sacrifices ou tourments ; comme le souligne Shimpach (2010 : 31), la plus grande diversité identitaire que mettent en scène les nouvelles séries est souvent représentée, non pas dans ses dimensions positives, mais bien dans la perte de pouvoir qu'elle symbolise pour l'homme blanc, désormais attaqué de toutes parts. Dans nombre de séries, la représentation de la crise et/ou du choc post-traumatique du personnage masculin ne donne en effet pas lieu, ultérieurement, à une transformation significative de son identité ou de ses choix de vie; la thématique semble servir davantage à montrer la souffrance émotionnelle des leaders, et donc à relativiser leurs avantages sociaux, plutôt qu'à encourager une transformation des scripts identitaires. Le pouvoir des hommes qui jouissent d'une hégémonie professionnelle et/ou de genre est ainsi minimisé.

De même, cette représentation est souvent associée à une mise en valeur de certaines qualités traditionnelles de la masculinité, telles que la force physique, le courage, le côté protecteur, ce qui permet simultanément la construction d'identités masculines transformées et revalorisées dans certaines de leurs caractéristiques les plus pérennes. 
Il y a dix ans déjà, Brenton J. Malin prédisait que les nouvelles masculinités mises en scène à la télévision états-unienne durant les années 2000 seraient caractérisées à la fois par une hypersensibilité et une force hypervirile (hypertoughness) (2005 : 179). Vue sous cet angle, la représentation plus fréquente des traumas physiques et psychologiques des hommes pourrait être interprétée comme une tentative de moderniser leur image, tout en évitant une transformation trop radicale. Comme le rappelait d'ailleurs E. Ann Kaplan, le concept de trauma est implicitement genré, ayant été habituellement associé aux hommes (2005: 19), surtout dans la théorie psychanalytique ; les études sur les traumas sont en effet principalement focalisées sur ceux vécus et/ou perpétrés par des hommes ${ }^{24}$.

De plus en plus fréquentes, les stratégies narratives employées pour représenter le monde intérieur de ces hommes (des scènes imaginées ou remémorées) ont également cette particularité d'être beaucoup moins systématiquement employées pour les personnages féminins. Par conséquent, le protagoniste masculin apparaît certes malheureux, mais il possède une gravitas, une vie intérieure représentée de manière plus empathique et complexe que chez les personnages féminins. En conclusion de son étude sur les séries états-uniennes, Amanda D. Lotz constatait d'ailleurs elle aussi cette grande complexité identitaire et narrative des séries centrées sur des hommes, par opposition à la plupart des œuvres centrées sur des protagonistes féminines : «Ceci étant dit, je me demande si les critiques de télévision et les chercheurs - et même les téléspectateurs - sont prêts à interpréter des personnages féminins aussi imparfaits et pleins de défauts que Walter White ou Dexter Morgan comme des individus et non pas comme une mise en cause du féminisme, des nouvelles femmes de carrière ou des mères $^{25}$.» (2014: 192) S'il est possible que les craintes de représentations sexistes aient freiné la construction d'identités complexes ou en crise chez les femmes, cette distinction a peut-être aussi à voir avec une conception implicitement différentielle des genres.

En interrogeant le paradoxe de la représentation télévisée des " masculinités en crise », le but n'est évidemment pas de la contester à tout prix ou d'encourager une dévalorisation totale des hommes dans les nouvelles fictions sérielles. L'objectif est plutôt de souligner que derrière l'apparence d'une représentation négative peuvent se cacher des mécanismes de valorisation implicites. La réception critique de ces récits, habituellement fort élogieuse ${ }^{26}$, corrobore cette hypothèse, puisqu'une telle représentation des hommes n'est souvent pas uniquement interprétée comme "réaliste »: elle est aussi souvent considérée comme un critère de "qualité » de l'œuvre. En effet, la crise masculine est fréquemment valorisée en tant que thématique centrale de la nouvelle qualité télévisuelle, comme l'a constaté le journaliste Brett Martin dans son récent ouvrage Difficult Men (2013), habilement traduit en français par Des hommes tourmentés (2014). Comme le rappelle Tania Modleski, la crise peut donc aussi servir paradoxalement à consolider l'hégémonie masculine : « ... il importe de considérer à quel point le pouvoir de l'homme se consolide précisément à travers des cycles de crise et de résolution, les hommes faisant ainsi face à la menace du pouvoir féminin en l'incorporant. ${ }^{27}$ Soulignons d'ailleurs que si les représentations sérielles véhiculent largement l'image d'une masculinité fragilisée, l'hégémonie des hommes au sein des trois industries télévisuelles étudiées est quant à elle encore intacte. Tant aux États-Unis, au Canada qu'au Québec, les hommes occupent encore la majorité des postes clés de la création télévisuelle (producteur exécutif, réalisateur, scénariste et directeur de la photo $^{28}$ ). Devant ces constats, on peut interpréter cette tendance télévisuelle 
comme à la fois une vision populaire de la réalité sociale et une conception récurrente et idéalisée du genre. Dans de nombreuses cultures, la masculinité a été définie dans sa dimension tragique et sacrificielle : les nouvelles séries télévisées sont peut-être ainsi une réactualisation, sous une forme transformée, de ce mythe de la masculinité en tant que crise.

Dans un contexte socioculturel où les modèles ouvertement positifs ou prospères de masculinité sont fréquemment qualifiés d'hégémoniques ou, du moins, paraissent suspects, la popularité de masculinités en crise pourrait ainsi être interprétée comme une stratégie nouvelle de valorisation oblique, notamment à travers le refus du happy end, d'une vision jugée naïve - féminine? - du monde et, plus encore, par la construction de nouvelles formes de distinction du féminin. Les masculinités dans ces fictions ne sont certes pas représentées comme étant parfaites ou idéales, mais elles apparaissent néanmoins valorisantes dans leur réaffirmation d'une complexité et d'une identité sacrifiée à l'idéologie du genre. Alors même que les conflits et rapports sociaux de sexe sont complexes et multiples, l'association prédominante des personnages masculins à une crise polymorphe semble ainsi révélatrice d'une conception différentielle des genres, d'une volonté tenace d'associer les hommes et les femmes à des récits polarisés, et donc de préserver la différence malgré les mutations récentes des normes sociales.

\section{BIBLIOGRAPHIE}

Allen Judith A. (2002), « Men Interminably in Crisis ? Historians on Masculinity, Sexual Boundaries, and Manhood », Radical History Review, nº 82, p. 191-207.

AtTAlah Paul (2013 [2007]), « A Usable History for the Study of Television », dans Tanner MIRRLEES et Joseph KISPAL-KOVACS (dir.), The Television Reader. Critical Perspectives in Canadian and US Television Studies, Don Mills, Oxford University Press, p. 78-92.

BADINTER Élisabeth (1992), XY De l'identité masculine, Paris, Éditions Odile Jacob.

BAKER Brian (2015), Contemporary Masculinities in Fiction, Film and Television, New York, Bloomsbury. BEARD William (2014), "Durham County Season 1 : Genre and Genre Subversion », Revue canadienne d'études cinématographiques, vol. 23, nº 2, p. 2-25.

BEATY Bart et Rebecca SULLIVAN (2006), Canadian Television Today, Calgary, University of Calgary Press.

BENASSI Stéphane (2004), « Existe-t-il un 'modèle français' de fiction policière télévisuelle à suite? », dans Pierre BEYLOT et Geneviève SELLIER (dir.), Les Séries policières, Paris, L'Harmattan, p. 111-126.

BEYNON John (2002), Masculinities and Culture, Philadelphie, Open University Press.

BLAIS Mélissa (2010), « Négocier la représentation de la tuerie du 6 décembre 1989 : une analyse du film Polytechnique », dans Mélissa BLAIS, Francis DUPUIS-DÉRI, Lyne KURTZMAN et Dominique 
PAYETTE (dir.), Retour sur un attentat antiféministe. École Polytechnique 6 décembre 1989, Montréal, les Éditions du remue-ménage, p. 101-107.

BLAIS Mélissa et Francis DUPUIS-DÉRI (2015), Le Mouvement masculiniste au Québec. L'antiféminisme démasqué ( $2^{\mathrm{e}}$ éd.), Montréal, Les éditions du remue-ménage.

BLY Robert (1990), Iron John. A book about men, Boston, Addison-Wesley.

BOISVERT Stéfany (2016), « Du dix-neuf au nineteen. Une étude comparative des versions québécoise francophone et canadienne anglophone de la série télévisée 19-2 ", Revue canadienne d'études cinématographiques, vol. 25, no 1 , p. 8-26.

BREDIn Marian, Scott HENDERson et Sarah A. MATHEson (dir.) (2012), Canadian Television: Text and Context, Ontario, Wilfrid Laurier University Press.

CHALVON-DEMERSAy Sabine (1994), Mille scénarios. Enquête sur l'imagination en temps de crise, Paris, Métailié.

CHozick Amy (2011), « A New Generation of TV Wimps », The Wall Street Journal. [En ligne] http:// online.wsj.com/news/articles/SB10001424052702304432304576371553682017000 [consulté le 7 octobre 2015].

CLARE Anthony (2001), On Men : Masculinity in Crisis, Londres, Arrow Books.

ConNell R.W. (2005), Masculinities, Berkeley, University of California Press.

Cowlishaw, Bridget Roussell (dir.) (2015), Masculinity in Breaking Bad : Critical Perspectives, Jefferson, McFarland \& Company.

COURTINE Jean-Jacques (2011), Histoire de la virilité. 3. La virilité en crise ? XX ${ }^{e}$-XXI siècle, Paris, Seuil. CURTIN Michael et Jane SHATTUC (2009), The American Television Industry, Londres, BFI/Palgrave Macmillan.

DE WASSEIGE Mathieu (2013), « Les séries télé des networks américains. Sujets de société, représentations sociales et balancement idéologique », Communication, vol. 32, no 1 [En ligne] https://communication.revues.org/4871

DE WASSEIGE Mathieu, avec la collaboration de Barbara DUPONT (2014), Séries télé US : l'idéologie prime time, Louvain-La-Neuve, Academia-L'Harmattan.

Dolezel Lubomír (1998), Heterocosmica, Baltimore/Londres, The Johns Hopkins University Press.

DRUick Zoë et Aspa Kotsopoulos (dir.) (2008), Programming Reality: Perspectives on English-Canadian Television, Waterloo, Wilfrid Laurier University Press.

DUBAR Claude (2010), La Crise des identités : L'interprétation d'une mutation, Paris, Presses universitaires de France.

DUPUIS-DÉRI Francis (2015), « Le 'masculinisme' : une histoire politique du mot », dans Mélissa BLAIS et Francis DUPUIS-DÉRI (dir.), Le Mouvement masculiniste au Québec. L'antiféminisme démasqué (2 éd.), Montréal, Les éditions du remue-ménage, p. 43-68.

DUPUIS-DÉRI Francis (2012), «Le discours de la 'crise de la masculinité' comme refus de l'égalité entre les sexes : histoire d'une rhétorique antiféministe », Recherches féministes, vol. 25, no 1 , p. 89-109.

EDWARDS Tim (2006), Cultures of Masculinity, New York, Routledge.

FALUDI Susan (1999), Stiffed: The Betrayal of the American Man, New York, W. Morrow and Co. 
FEASEY Rebecca (2008), Masculinity and Popular Television, Edinburgh, Edinburgh University Press.

FISKE John (2011 [1987]), Television Culture, New York, Routledge.

FRATICELli Rina (2015), Women in View on Screen : October 2015 Report, Toronto, Women in View. [En ligne] http://trends.cmf-fmc.ca/media/uploads/reports/Women-in-View-on-screen-EN.pdf GuionNET Christine et Erik Neveu (2009), Féminins/Masculins. Sociologie du genre, Paris, Armand Colin.

HAYEUR Isabelle et Sophie BISSONNETTE (2016), La Place des créatrices dans les postes clés de création de la culture au Québec, Réalisatrices équitables [En ligne] http://realisatrices-equitables.com/wpcontent/uploads/2016/06/rapport-la-place-des-creatrices-12-juin-2016.pdf

HEARTFIELD James (2002), « There is No Masculinity Crisis », Genders, nํ 35. [En ligne] http:// www.genders.org/g35/g35_heartfield.html [consulté le 7 octobre 2015].

HoRRocks Roger (1994), Masculinity in Crisis, New York, St. Martin’s Press.

Jost François (2015), Les Nouveaux méchants. Quand les séries américaines font bouger les lignes du Bien et du Mal, Montrouge, Bayard.

KAPLAN E. Ann (2005), Trauma Culture: The Politics of Terror and Loss in Media and Literature, New Brunswick, Rutgers University Press.

Kimmel Michael (2013), Angry White Men : American Masculinity at the End of an Era, New York, Nation Books.

Kimmel Michael (2012), Manhood in America : A Cultural History ( $3^{\mathrm{e}}$ éd.), New York, Oxford University Press.

Kimmel Michael S. (1987), « The Contemporary 'Crisis' of Masculinity in Historical Perspective », dans Harry BROD (dir.), The Making of Masculinities: The New Men's Studies, Boston, Allen \& Unwin, p. 121-153.

LoTz Amanda D. (2014), Cable Guys : Television and Masculinities in the 21st Century, New York, New York University Press.

MAC MAHON Anthony (2005), « Lectures masculines de la théorie féministe : la psychologisation des rapports de genre dans la littérature sur la masculinité », L'Homme et la société, vol. 158, n 4 , p. 27-51.

MACÉ Éric (2015), L’Après-patriarcat, Paris, Éditions du Seuil.

MACÉ Éric (2006), La Société et son double : Une journée ordinaire de télévision, Paris, INA/Armand Colin.

MACÉ Éric (2003), « Le conformisme provisoire de la programmation », Hermès, nº 37, p. 127-135.

MACINNES John (1998), The End of Masculinity, Buckingham/Philadelphie, Open University Press.

MAIGRET Éric et Éric MACÉ (2005), Penser les médiacultures. Nouvelles pratiques et nouvelles approches de la représentation du monde, Paris, INA/Armand Colin.

MaLiN Brenton J. (2005), American Masculinity Under Clinton: Popular Media and the Nineties 'Crisis of Masculinity', New York, Peter Lang.

MARTIN Brett (2014), Des hommes tourmentés : le nouvel âge d'or des séries : des Soprano et The Wire à Mad Men et Breaking Bad, Paris, Éditions de la Martinière. 
MARTIN Brett (2013), Difficult Men. Behind the Scenes of a Creative Revolution : From The Sopranos and The Wire to Mad Men and Breaking Bad, New York, The Penguin Press.

MAUGUE Annelise (1987), L'Identité masculine en crise au tournant du siècle, Paris, Éditions Rivages. MCCABE Janet et Kim AKASS (dir.) (2007), Quality TV : Contemporary American Television and Beyond, New York, I.B. Tauris.

MigNER-LAURIN Anne et Anouk BÉLANGER (2012), Les Réalisatrices du petit écran. [1952-2012] 60 ans de télévision québécoise, Montréal, Bibliothèque et archives Canada, Université du Québec à Montréal. MirRleEs Tanner et Joseph KISPAL-Kovacs (dir.) (2013), The Television Reader : Critical Perspectives in Canadian and US Television Studies, Don Mills, Oxford University Press.

MitTell Jason (2015), Complex TV : The Poetics of Contemporary Television Storytelling, New York/ Londres, New York University Press.

MODLESKI Tania (1991), Feminism Without Women: Culture and Criticism in a 'Postfeminist' Age. New York, Routledge.

MoINE Raphaëlle (2008), Les Genres du cinéma (2e éd.), Paris, Armand Colin.

MOLINIER Pascale (2004), « Déconstruire la crise de la masculinité », Mouvements, no 31, p. 24-29.

MORIN Edgar (2008), L'Esprit du temps (nouvelle éd.), Paris, Armand Colin/INA.

MoRIN Edgar (1975), L’Esprit du temps. Tome 2 : Nécrose, Paris, Bernard Grasset.

Moss Mark (2011), The Media and the Models of Masculinity, Lanham, Lexington Books.

PERREUR Nathalie (2011), « La néo-série, arène d'évaluation culturelle d'une société américaine en crise », Réseaux, n 165, p. 83-108.

PERREUR Nathalie (2007), « La 'néo-série’ policière et judiciaire américaine, miroir des anxiétés américaines ", Médiamorphoses, $n^{\circ}$ 3, p. 90-93.

PetrowsKi Nathalie (2011), «Les filles de 19-2 », La Presse. [En ligne] http://www.lapresse.ca/arts/ television/201112/09/01-4476398-les-filles-de-19-2.php [consulté le 1 octobre 2015].

PRoulX Serge et Danielle BÉLANGER (2001), « La représentation des communautés immigrantes à la télévision francophone du Québec. Une opportunité stratégique », Réseaux, vol. 19, nº 107, p. $117-145$

RALPH J. BUNCHE CENTER FOR AFRICAN AMERICAN STUDIES (2015), 2015 Hollywood Diversity Report : Flipping the Script, Los Angeles, UCLA.

RAUCH André (2000), Crise de l'identité masculine, 1789-1914, Paris, Hachette.

REHM Félix (2014), « Desperate Housemen », artpress 2 « Séries télévisées. Formes, fabriques, critiques », no 32 , p. 65-70.

RoBInson Sally (2000), Marked Man : White Masculinity in Crisis, New York, Columbia University Press.

Rosin Hanna (2012), The End of Men and the Rise of Women, New York, Riverhead Books.

Rosin Hanna (2011), « Primetime's Looming Male Identity Crisis », The Atlantic. [En ligne] http:// www.theatlantic.com/entertainment/archive/2011/09/primetimes-looming-male-identitycrisis/244692/\#slide1 [consulté le 7 octobre 2015]. 
Rosin Hanna (2010), « The End of Men », The Atlantic. [En ligne] http://www.theatlantic.com/ magazine/archive/2010/07/the-end-of-men/308135/ [consulté le 1 octobre 2015].

SÉRISIER Pierre, Marjolaine BOUTET et Joël BASSAGET (2011), Sériescopie. Guide thématique des séries télé, Paris, Ellipses.

SEPINWALl Alan (2015), The Revolution Was Televised: How The Sopranos, Mad Men, Breaking Bad, Lost, and Other Groundbreaking Dramas Changed TV Forever (2e éd.), New York, Touchstone.

SHIMPACH Shawn (2010), Television in Transition: The Life and Afterlife of the Narrative Action Hero, Malden/Oxford, Wiley-Blackwell.

SHWEDER Richard A. (1994), "What Do Men Want? A Reading List for the Male Identity Crisis », The New York Times. [En ligne] http://www.nytimes.com/1994/01/09/books/what-do-men-wanta-reading-list-for-the-male-identity-crisis.html?pagewanted=all [consulté le 2 octobre 2015].

STEENBERG Lindsay et Yvonne TASKER (2014), « North of Quality? 'Quality' Television and the Suburban Crimeworld of Durham County », dans Jeannette SLONIOWSKI et Marilyn ROSE (dir.), Detecting Canada : Essays on Canadian Crime Fiction, Television, and Film, Waterloo, Wilfrid Laurier University Press, p. 257-274.

TASKER Yvonne et Diane NEGRA (dir.) (2007), Interrogating Postfeminism : Gender and the Politics of Popular Culture, Durham, Duke University Press.

Tremblay Gilles et Pierre L'Heureux (2010), « La genèse de la construction de l'identité masculine ", dans Jean-Martin DESLAURIERS et al. (dir.), Regards sur les hommes et les masculinités, Québec, Presses de l'Université Laval, p. 91-123.

WALSH Fintan (2010), Male trouble : masculinity and the performance of crisis, New York, Palgrave Macmillan.

WATSON Elwood (2009), Pimps, wimps, studs, thugs and gentlemen : essays on media images of masculinity, Jefferson, McFarland \& Co.

Welzer-LANG Daniel et Chantal ZaOuche GAUdRon (dir.) (2011), Masculinités : état des lieux, Toulouse, Érès.

WHITEHEAD Stephen M. (2002), Men and Masculinities : Key Themes and New Directions, Malden, Polity.

\section{NOTES}

1. Bien que le Québec fasse partie du Canada, sa production est habituellement analysée séparément. Tout d'abord pour une raison linguistique : le Québec est une province à dominante francophone, reconnue comme une nation distincte au sein du Canada depuis 2006, alors que le reste du pays est à dominante anglophone. Par conséquent, les productions télévisuelles québécoises sont produites en français, alors que celles s'adressant plus largement à un auditoire canadien sont majoritairement produites en anglais. Cette distinction entre les productions télévisuelles québécoises et canadiennes est fréquemment préconisée en études télévisuelles (Beaty et Sullivan, 2006; Bredin, Henderson et Matheson, 2012; Druick et Kotsopoulos, 2008 ; Mirrlees et Kispal-Kovacs, 2013; Moss, 2011). Par ailleurs, soulignons que les productions québécoises sont massivement regardées dans la province, alors que les Canadiens anglais regardent très peu leurs productions locales (Druick et Kotsopoulos, 2008 : 1-2). Pour un résumé des distinctions entre les industries télévisuelles québécoise et canadienne, voir Attalah, 2013. 
2. "Their condition strikes me as symptomatic of a male malaise - what I loosely call male autism - a state of being cut off from natural feelings and expressiveness and contact with others. » (107) ma traduction. 3. "These elegiac accounts tend to be more sympathetic, concerned with men as victims of circumstances beyond their control [...]. » (2) ma traduction.

4. Le mouvement mythopoétique des hommes est apparu principalement aux États-Unis en réaction à la seconde vague féministe et s'appuie, globalement, sur le mythe d'une masculinité profonde et originelle que les hommes devraient tenter de retrouver. Le monde moderne aurait en effet éloigné les hommes de leur véritable «identité» ou "nature ", voire les aurait dangereusement féminisés, ce qui expliquerait leurs troubles identitaires actuels. En participant à des retraites entre hommes ou à divers rituels, les hommes pourraient retrouver leur masculinité. Le mouvement valorise ainsi une conception profondément essentialiste des genres, centrée sur une solide distinction des hommes et des femmes. Le célèbre ouvrage Iron John de Robert Bly (1990) est exemplaire de cette mouvance.

5. "I don't think I realized it until this moment, but it must be hard being a man, too. » ma traduction.

6. Contrairement aux chaines premium (payantes) (HBO, Showtime, Starz, etc.), qui se financent par les abonnements à la carte, les chaines du câble de base (basic cable) (AMC, FX, USA Network, etc.) sont vendues sous forme de bouquet (forfait) et s'appuient sur deux sources de revenus : la vente d'espaces publicitaires et les frais d'abonnement. Les épisodes des séries du câble de base (par exemple, Breaking Bad, Mad Men) sont donc entrecoupés de segments publicitaires, contrairement aux fictions des chaines premium (The Sopranos, True Detective, Ray Donovan, etc.), exemptes d'interruptions.

7. «But you're not a man, Lester. You're not even half a man » ma traduction.

8. « Grow a pair of balls. » ma traduction.

9. Le groupe de policiers et policières sur lequel se concentre la série Flashpoint se nomme la «Strategic Response Unit » (unité stratégique d'intervention), une dénomination fictive équivalant à un groupe d'intervention d'urgence (Emergency Task Force). Ce groupe a été formé afin de pouvoir intervenir lors de situations à haut risque où la vie de personnes est directement menacée, notamment des prises d'otage ou des alertes à la bombe.

10. "You know what else I see? All the calls we didn't get to in time. [...] You're not the only one who sees ghosts, Danny. » ma traduction.

11. «He's not broken, he's cracked " ma traduction.

12. Nous employons le concept d'hégémonie (masculinité hégémonique) tel qu'il fut théorisé par R.W. (Raewyn) Connell: «La masculinité hégémonique peut être définie comme une configuration de pratiques genrées qui incarne aujourd'hui la réponse socialement acceptée au problème de la légitimité du patriarcat, lequel garantit (ou est supposé garantir) la position dominante des hommes et la subordination des femmes. » ma traduction : «Hegemonic masculinity can be defined as the configuration of gender practice which embodies the currently accepted answer to the problem of the legitimacy of patriarchy, which guarantees (or is taken to guarantee) the dominant position of men and the subordination of women. » (2005:77)

13. Pour une étude plus exhaustive de cette œuvre, voir notre article présentant une analyse comparative des versions québécoise francophone et canadienne anglophone de la série télévisée 19-2 (2016).

14. Bien que d'autres définitions du terme soient possibles, le post-féminisme a néanmoins souvent des connotations antiféministes: "Même si le féminisme est aujourd'hui très dynamique, déclarer qu'il est archaïque et prétendre qu'il est mort sont autant de façons de discréditer ce mouvement et de miner sa légitimité. » (Dupuis-Déri, $2015: 130$ )

15. Nous reprenons ici la distinction proposée par Tim Edwards (2006: 6) entre une «crise extérieure " (crisis from without), laquelle fait référence à la position menacée des hommes en société, à la perte de leurs pouvoirs et privilèges en tant que classe, et une "crise intérieure ", 
psychologique (crisis from within), laquelle renvoie plutôt au mal-être ou à toute autre forme de dysfonction identitaire entrainant des souffrances émotionnelles.

16. Dans son ouvrage Mille scénarios (1994), Chalvon-Demersay propose une synthèse analytique de nombreux projets de fiction télévisuelle qui avaient été soumis dans le cadre d'un concours pour France Télévisions.

17. "The rhetoric of crisis is flexible enough to accommodate a range of narratives driven by competing investments and intentions. " (Robinson, $2000: 11)$ ma traduction.

18. Tout en reconnaissant que les séries ont une orientation idéologique et opèrent une clôture du sens, il importe néanmoins de rappeler qu'une œuvre est toujours polysémique (Fiske, 2011) ; par conséquent, les interprétations d'une même série peuvent varier. Par exemple, si certains téléspectateurs pourront interpréter le récit d'un homme en crise comme une critique des modèles traditionnels de genre qui encourage une transformation des identités, d'autres au contraire pourront plutôt y voir la célébration d'une masculinité immuable, condamnée à être en crise ou "allergique» au bonheur. La série Breaking Bad est un exemple bien connu de polarisation interprétative: par la transformation radicale du personnage principal qu'elle opère, la série a été interprétée par certains téléspectateurs selon une vision masculiniste, c'està-dire comme une célébration du passage d'une masculinité subordonnée à une masculinité hégémonique (comme en témoignent les nombreux sites Internet haineux à l'endroit du personnage de Skyler, l'épouse de Walter White, traitée de «salope » ou de «bitch», décrite comme une femme castratrice nuisant au cheminement du « héros ») ; d'autres au contraire l'ont interprétée comme une critique de la culture masculine traditionnelle et, surtout, des conséquences désastreuses (violence, meurtres, destruction de la cellule familiale) auxquelles mène la masculinité hégémonique. Pour un résumé des réactions haineuses contre le personnage de Skyler dans Breaking Bad et de ses différentes interprétations, voir Cowlishaw (2015 : 132-138). 19. Songeons au personnage de Ray Donovan, digne représentant d'une masculinité hégémonique, valorisé pour ses compétences professionnelles, sa force physique et son charme, mais également montré comme profondément malheureux, alcoolique, avec des comportements fréquemment autodestructeurs. Un cas exemplaire est également celui de Don Draper (Mad Men), dont le cheminement identitaire, marqué par une dépression latente, se conclura, au dernier épisode, par sa participation à un groupe d'aide psychologique (seminar) au sein d'une retraite hippie. Un des hommes y parle alors de son sentiment persistant d'avoir une vie restrictive et insatisfaisante, de n'être rien pour personne, d'être seul, de ne pas ressentir l'amour. Visiblement ému et se reconnaissant dans cette description, Don va alors l'étreindre et pleure avec lui.

20. S'appuyant sur un article de Rick Altman, Raphaëlle Moine utilise ce terme de "genrification » en référence aux processus de construction des genres narratifs au cinéma (2008), qui s'opère selon " une dialectique constante entre la consolidation et la dissémination des catégories » (Altman, cité dans Moine 2008 : 135). Il nous semble donc pertinent d'employer ce terme afin de mettre en lumière la performativité du genre (gender) qui est à l'œuvre lors de la création de genres narratifs. Nous employons donc ici le néologisme de "genrification » afin de définir ce double processus à travers lequel l'élaboration de genres télévisuels (narratifs) s'accompagne d'un processus d'élaboration et de reproduction, à travers les récits, d'une distinction binaire et sexuée associée à une « somme d'attributs, d'attentes, de rôles, de statuts, de traits psychiques et comportementaux culturels et sociaux qui définissent ce qui est désigné, selon les contextes, comme masculin ou féminin " (ce qu'Éric Macé appelle la "genration») (Macé, 2015 : 17-18). Dans son ouvrage, Fiske (2011) range ainsi les fictions télévisuelles en genres "féminin» et «masculin»; il affirme que les caractéristiques (thématiques, sémantiques, syntaxiques) des genres narratifs du médium (les soap operas, les séries d'action, etc.) sont établies en fonction d'attentes conformistes concernant les goûts et identités de genre du public visé. 
21. Breaking Bad est probablement l'une des séries contemporaines où les injonctions au respect d'une norme masculine sont les plus récurrentes. À titre d'exemple, rappelons que tous les actes criminels du personnage principal y sont justifiés par la nécessité pour un homme de subvenir aux besoins financiers de sa famille, comme le rappellera d'ailleurs clairement Gus Fring, le chef d'un cartel de drogue : "[...] a man provides. And he does it even when he's not appreciated or respected or even loved. He simply bears up, and he does...because he's a man.» [S03XE05] («[...] un homme subvient aux besoins de sa famille. Et il le fait, même s'il n'est pas apprécié, respecté, ou même aimé. Il supporte simplement cette situation, et il le fait... parce qu'il est un homme.»ma traduction.

22. Il y aurait également un lien à faire avec les travaux de Mathieu de Wasseige $(2013,2014)$ concernant le balancement idéologique de nombreuses séries télévisées. Selon cet auteur, tout en adoptant une position idéologique centrale (endiguement idéologique), les séries octroient souvent des concessions idéologiques, ce qui permettrait de satisfaire un public plus diversifié.

23. Il est toutefois important de préciser que le personnage de John est homosexuel, une caractéristique plus rare lorsqu'il est question de la « masculinité en crise ».

24. "Their neglect is partly due to the implicit gendering of trauma studies, such that traumas of (and perpetrated by) men have been a main focus. » (Kaplan, 2005 : 19) ma traduction.

25. "That said, I still wonder whether television critics and scholars - and audiences, for that matter - are ready to see female characters that are as deeply flawed as Walter White and Dexter Morgan as individuals and not as indictments of feminism, contemporary career women, and mothers. » ma traduction.

26. Plusieurs des séries états-uniennes qui ont été abordées ici sont associées à la Quality TV (Martin, 2013 ; McCabe et Akass, 2007 ; Sepinwall, 2015). De même, au Québec, les journalistes se sont grandement intéressés aux œuvres que nous avons présentées et les ont souvent associées à une nouvelle «qualité » de la télévision québécoise. Au Canada anglais, nous retrouvons une fascination similaire pour les œuvres centrées sur des hommes tourmentés ou en crise. Beard (2014) et Steenberg et Tasker (2014) ont documenté l'intérêt international pour la série Durham County, considérée comme un exemple d'une nouvelle qualité télévisuelle canadienne.

27. «[...] we need to consider the extent to which male power is actually consolidated through cycles of crisis and resolution, whereby men ultimately deal with the threat of female power by incorporating it. » (Modleski, 1991 : 7) ma traduction.

28. Au Québec, en 2010-2011, les hommes ont réalisé plus de $80 \%$ des émissions les plus populaires (Migner-Laurin et Bélanger, 2012 : 48). De plus, ceux-ci ont réalisé de 57 à $80 \%$ des dramatiques télévisées; sur le pourcentage restant, les femmes ayant réalisé seules une production sont très rares, voire inexistantes sur certaines chaines: celles-ci sont plus fréquemment intégrées à une équipe mixte. Selon un rapport de Réalisatrices équitables (Hayeur et Bissonnette, 2016:10), la parité est toutefois atteinte dans les postes de scénarisation. Au Canada anglais, l'étude Women in View on Screen (Fraticelli, 2015) arrive à un constat similaire : de tous les projets financés par le Fonds des médias du Canada (FMC) en 2012-2013, aucun n'avait été réalisé par une femme. Plus généralement, au sein des positions créatives clés, les hommes ont obtenu $74 \%$ des postes, contre $26 \%$ pour les femmes (12). L'étude montre aussi que les femmes ont davantage de chances d'être écartées des projets disposant des plus gros budgets (16). En ce qui concerne la télévision états-unienne, le Hollywood Diversity Report de 2015 (Ralph J. Bunche Center for African American Studies) révèle une inégalité systémique entre hommes et femmes en ce qui concerne l'octroi de postes créatifs ou décisionnels : en 2012-2013, les hommes ont obtenu la majorité des rôles principaux dans les fictions télévisuelles des grands networks (54\%), et $58 \%$ sur les chaines câblées (20). Qui plus est, plus de $70 \%$ des créateurs de séries $(71.2 \%$ sur les networks, 77.4 \% sur les chaines câblées) et des dirigeants de l'industrie jouissant du pouvoir décisionnel (TV executives) étaient des hommes $(71 \%)(23,29)$. En ce qui concerne les séries étudiées ici, l'identité des créateurs et/ou scénaristes ne permet toutefois pas de postuler un lien de cause à effet entre leur genre et la présence de la thématique de la «masculinité en crise ». 
Certaines séries ont en effet été créées et écrites par un ou des homme(s) seulement, d'autres par des femmes uniquement, d'autres encore par une équipe mixte.

\section{RÉSUMÉS}

Cet article aborde le sujet de la «masculinité en crise » en tant que norme représentationnelle dans de nombreuses séries télévisées dramatiques contemporaines en Amérique du Nord (ÉtatsUnis, Canada, Québec). Cette thématique est souvent associée de manière quasi exclusive aux chaînes câblées états-uniennes, alors que sa popularité est beaucoup plus transnationale. Pour cette raison, cette thématique doit être appréhendée en tant que tendance interprétative dominante des genres au XXI ${ }^{\mathrm{e}}$ siècle, laquelle s'appuie sur une perception sociale concernant la « crise » des identités masculines. À la suite de la présentation d'une typologie des hommes « en crise " dans un corpus nord-américain, des pistes interprétatives seront proposées afin de comprendre les significations de ce phénomène culturel. L'hypothèse principale est qu'au-delà de la vision négative colportée par de telles représentations, la thématique de la crise peut aussi être interprétée comme une stratégie oblique de valorisation de la masculinité et de réaffirmation d'une différence fondamentale des genres.

This paper focuses on the "masculinity crisis » as a popular theme in many contemporary TV dramas in North America (United States, Canada, Quebec). This narrative trend, far from being restricted to cable TV series in the US, is transnational. The popularity of «narrative crises " (Lotz, 2014) in male-centered TV dramas reflects a contemporary social concern with changing gender norms. In order to better understand the meanings behind this narrative trend, we will define different types of "male characters in crisis " and try to determine their narrative functions. This will lead us to the conclusion that even though the «masculinity crisis » is usually cast in a negative light, this theme may also have a positive function: the representation of masculine crises could indeed be interpreted as a validation strategy, a peculiar way to idealize new masculinities and new forms of gender differentiation.

\section{INDEX}

Mots-clés : masculinités, crise, identité, séries télévisées, dramatiques, États-Unis, Canada, Québec

Keywords : masculinities, masculinity in crisis, identity, TV series, dramas, United States, Canada, Quebec

\section{AUTEUR}

\section{STÉFANY BOISVERT}

Stéfany Boisvert est chercheuse postdoctorale au sein du département d'histoire de l'art et d'études en communication de l'université McGill et membre du centre de recherche Media@McGill. Elle participe actuellement à plusieurs projets de recherche portant sur la télévision, le cinéma, la sérialité et les études de genre. Sa recherche doctorale portait sur les 
discours identitaires masculins dans les fictions télévisuelles nord-américaines contemporaines (bourse d'études supérieures du Canada Joseph-Armand-Bombardier). Elle a notamment publié dans la Revue canadienne d'études cinématographiques ainsi que dans Télévision, Cinéma \& Cie et Screen. 